\title{
Spatiotemporal regulation of fibroblast growth factor signal blocking for endoderm formation in Xenopus laevis
}

\author{
Sang-wook Cha', Jong-Woo Lee ${ }^{3}$, \\ Yoo-seok Hwang ${ }^{4}$, Jeong-Pil Chae', \\ Kwon Moo Park', Hee Jung Cho', \\ Dong Sun Kim', Yong Chul Bae ${ }^{2}$ \\ and Mae Ja Park ${ }^{1,5}$ \\ ${ }^{1}$ Department of Anatomy \\ School of Medicine \\ ${ }^{2}$ Department of Oral Anatomy \\ School of Dentistry \\ Kyungpook National University \\ Daegu 700-412, Korea \\ ${ }^{3}$ Department of Urology \\ Gumi Cha Hospital \\ Gumi 730-728, Korea \\ ${ }^{4}$ Laboratory of Molecular Genetics \\ $\mathrm{NICHD}, \mathrm{NIH}$, Bethesda, Maryland, USA \\ ${ }^{5}$ Corresponding author: Tel, 82-53-420-4802; \\ Fax, 82-53-426-9085; E-mail, mjpark@knu.ac.kr \\ DOI 10.3858/emm.2008.40.5.550
}

Accepted 24 June 2008

Abbreviations: FGF, fibroblast growth factor; GFP, green fluorescence protein; MBT, midblastula transition; MMR, Marc's modified Ringer

\begin{abstract}
We have previously shown that the inhibition of fibroblast growth factor (FGF) signaling induced endodermal gene expression in the animal cap and caused the expansion of the endodermal mass in Xenopus embryos. However, we still do not know whether or not the alteration of FGF signaling controls embryonic cell fate, or when FGF signal blocking is required for endoderm formation in Xenopus. Here, we show that FGF signal blocking in embryonic cells causes their descendants to move into the endodermal region and to express endodermal genes. It is also interesting that blocking FGF signaling between fertilization and embryonic stage 10.5 promotes endoderm formation, but persistent FGF signaling blocking after stage 10.5 restricts endoderm formation and differentiation.
\end{abstract}

Keywords: embryonic development; endoderm; fibroblast growth factors; SU 5402; Xenopus

\section{Introduction}

Embryonic cells that have just finished cell cleavage choose their fate among one of three germ layers depending on their maternally determined extraand intracellular environment. This process, called germ layer specification, is one of the rate-limiting events in vertebrate embryogenesis. Through germ layer specification, the equatorial and the vegetal regions of Xenopus embryos are specified as mesoderm and endoderm, respectively (Heasman, 1997; Munoz-Sanjuan and H-Brivanlou, 2001; Stemple, 2001). A large number of studies have been performed to study germ layer specification, but the underlying mechanism and molecular determinants of the process are still important and unanswered questions in developmental biology.

In Xenopus, the signaling pathways of endoderm formation are activated by VegT, a maternally encoded T-box transcription factor. VegT acts via nodal signaling and is upstream of endodermspecific genes such as MIXs, Xsox17, endodermin, and Cerberus (Clements et al., 1999, 2003; Dale, 1999; Xanthos et al., 2001; Heasman, 2006). In addition, several major pathways including canonical Wnt signaling, GATA factors, and Notch signaling also activate endoderm specific genes, strongly suggesting the complexity of the molecular hierarchy in endoderm formation (Agius et al., 2000; Patient and McGhee, 2002; Hilton et al., 2003; Vincent et al., 2003; Kofron et al., 2004; Wardle and Smith, 2006).

It has been well known that FGF signaling plays a role as an endogenous mesoderm inducer and a priming factor in early neurogenesis of the vertebrate embryo (Amaya et al., 1993; Cornell et al., 1995; Ciruna and Rossant, 2001; Delaune et al., 2005). However, the effects of deprivation of FGF signaling on the embryo had not been noticed until we showed the effects of FGF signaling deprivation on endoderm formation (Heasman, 1997; Cha et al., 2004). In our previous study, blocking FGF signaling induced endodermal gene expression in animal cap explants and increased endodermal mass (Cha et al., 2004). The expanded endodermal mass seemed to be caused by endodermalization of mesoectodermal cells following FGF signal blockade, since the volume of endodermal tissue was changed dramatically depending on the presence or absence of FGF signal. If so, this 
means that the presence or absence of FGF signaling in embryonic cells could indeed alter their fate. The signal would divert the embryonic cells away from the place where they are supposed to be. It would be interesting to know whether blocking FGF signal converts the fate of embryonic cells into endoderm and when the FGF signal blockade is required for proper endoderm formation.

In this study, we have examined the possibility of fate alteration of embryonic cells following FGF signaling blockade and defined the critical time window of FGF signaling blockade for endoderm formation. We show for the first time that blocking FGF signal in embryonic cells caused their descendants to move into endodermal territory by using cell lineage tracing methods. Furthermore, we show that blocking FGF signal with SU5402 soaked beads induced the ectopic expression of endodermal genes in the surrounding presumptive ectodermal cells. Timed inhibition of FGF signaling showed that the blockade during the period from fertilization to stage 10.5 accelerated endoderm formation but that the prolonged blockade after stage 10.5 suppressed the maintenance and differentiation of endoderm. All these results suggest that blocking FGF signal in vegetal cells before stage 10.5 makes them adopt an endodermal fate and explains why the FGF signaling pathway is not active in the vegetal embryonic cells during this limited time of Xenopus embryogenesis.

\section{Materials and Methods}

\section{Xenopus embryo manipulation}

Xenopus laevis were obtained by in vitro fertilization. Developmental stages were characterized according to standards from Nieuwkoop and Faber (Nieuwkoop and Faber, 1994). Embryos were injected with mRNA or DNA and manipulated as described in the figure legends.

\section{RT-PCR analysis with animal caps and whole embryo}

Total RNA was extracted from whole embryos or cultured explants with TRIzol reagent (MRC) following the manufacturer's instructions (http://www. mrcgene.com/tri.htm). RT-PCR was performed with a Revertaid cDNA synthesis kit (MD). Primers used in this study are summarized in Table 1.

\section{Lineage tracing assay}

In order to identify cell lineage, we used nuclear localized $\beta$-galactosidase (n- $\beta$-gal) or green fluorescence protein (GFP) mRNA as a tracer (100 $\mathrm{pg} /$ whole embryo). After co-injecting DN-FR or $\mathrm{DN}-\mathrm{BR}$ mRNA with $\mathrm{n}$ - $\beta$-gal or GFP into a single blastomere at the 32-cell stage embryos, embryos were cultured to the desired stage. The embryos were imaged with a Leica MZ16FA stereomicroscope.

\section{Tissue recombination assay}

Animal cap explants were dissected at stages $8.5 \sim 9$ and cultured in 30\% Marc's modified Ringer (MMR) containing $50 \mathrm{~g} / \mathrm{ml}$ gentamycin until the desired stages for further analysis as described in the figure legends. SU5402 (10 M) (Calbiochem Inc. Germany), a chemical inhibitor of FGFR1 $\alpha$, was used to block FGF signaling (Mohammadi et al., 1997). In the conjugation experiments, recombinants were cultured for $40 \mathrm{~min}$ at $16^{\circ} \mathrm{C}$ to heal the torn ends of the tissue explants before being transferred to fresh $30 \%$ MMR media.

Table 1. Primers used in RT-PCR.

\begin{tabular}{lll}
\hline & Upstream primer (5'-3') & Downstream primer (5'-3') \\
\hline m-Actin & gctgacagaatgcagaag & ttgcttggaggagtgtgt \\
N-CAM & cacagttccaccaaatgc & ggaatcaagcggtacaga \\
IFABP & gccttgatggaacttggaa & ctgtaggaaccaggcaccat \\
Globin & gcctacaacctgagagtgg & caggctggtgaggctgccc \\
Xlhbox8 & cctacagcaacccttggta & gggctcttgtgtaggctgtc \\
Mixer & caccagcccagcacttaacc & caatgtcacatcaactgaag \\
Endodermin & agcagaaaatggcaaacacac & ggtctttaatggcaacaggt \\
Xsox17a & aggtgaagaggatgaagagg & agagcctggcaagtacatct \\
EF1 $\alpha$ & cctgaaccacccaggccagattggtg & gagggtagtcagagaagctctccacg \\
Darmin (in situ $)$ & atcgatatgttccgcttgttcatca & ctcgagagttcaggaagatgttgaa \\
Enf $($ in situ $)$ & gaattcatggcatccagcaggtcac & ctcgagtctttagcgcaactgagct \\
Endodermin $(i n$ situ $)$ & tgaggaggatacacgaacca & agtgagagagtgattcgacc \\
\hline
\end{tabular}




\section{Whole mount and hemi-sectioned in situ hybridization}

Whole mount in situ hybridization was performed as previously described with some modifications. BM purple AP substrate (Roche, Germany) was used for staining (Sive et al., 2000). For in vitro riboprobe transcription, the Megascript kit (Ambion, TX) was used following the manufacturer's ins- tructions.

\section{Histology}

For histological characterization, embryos were sectioned using a Leica microtome after X-gal staining. To enhance the contrast, we de-paraffinized sections and imaged them using a DIC objective lens
A

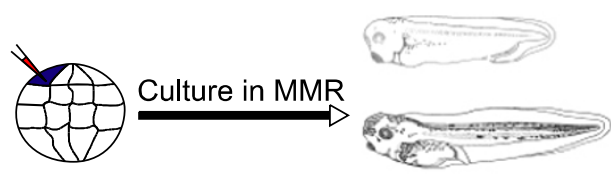

n-gal

$\mathrm{n}$-gal + DNFR mRNA

A tier
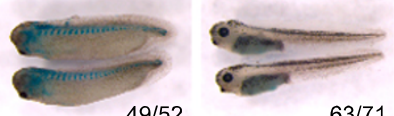

B tier
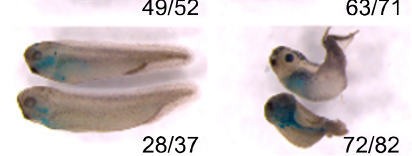

C tier
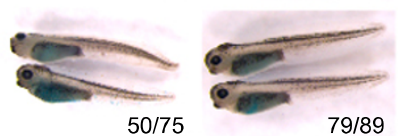

D tier
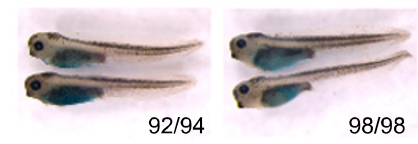

B

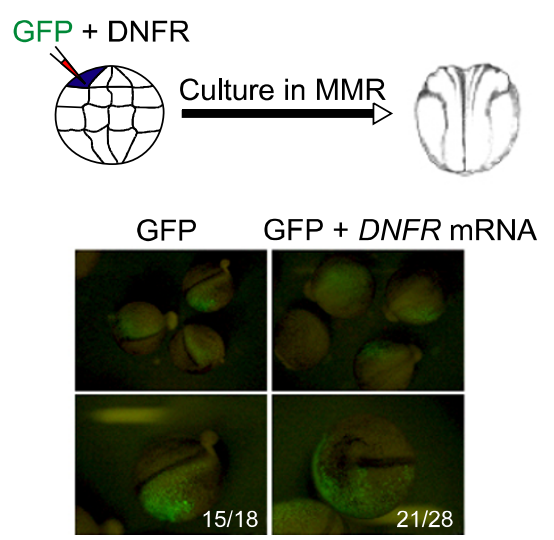

C

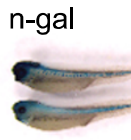

n-gal + DNFR



n-gal + eFGF

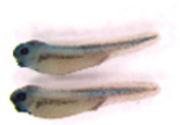

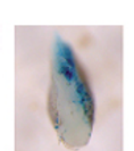

n-gal + DNBR

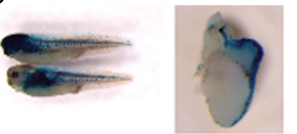

n-gal + DNBR + DNFR
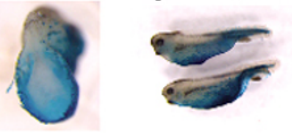

n-gal + DNFR + WTFR
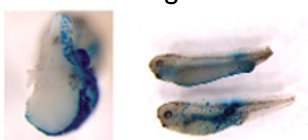
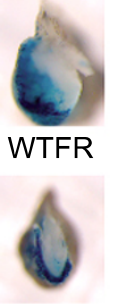

D

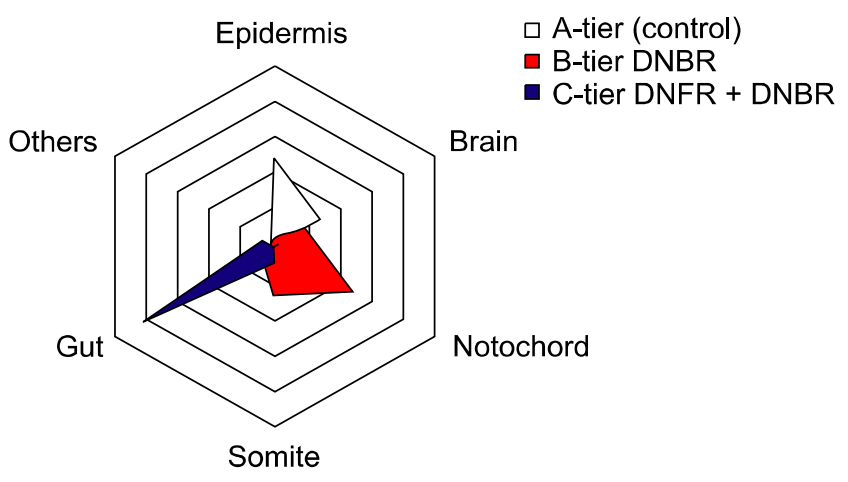

Figure 1. Effects of blocking FGF signal following DN-FR injection into a single dorsal blastomere in the $A, B, C$, or $D$ tiers at the 32-cell stage. $\mathrm{n}$ - $\beta$-galactosidase was used as a tracer. Tadpoles were fixed and stained for $\beta$-galactosidase at stage 42 . Two representative embryos are shown above. (A) Resultant morphological phenotype was normal when DN-FR was injected into a single blastomere in the A, C, or D tiers. However, embryos injected with DN-FR in the B tier showed a severely malformed phenotype, including a curved back and shortened tail. Interestingly, the data showed the trend that descendants of the DN-FR injected blastomere in A-, C-, and D-tier localized mostly in the endodermal territory. These data are summarized in Table 2. (B) Descendants of a single blastomere in the A tier co-injected with DN-FR and GFP were traced in the endodermal area (Table S1). (C) After DN-FR injection into a single blastomere of each 32-cell stage embryo, their descendants were traced at stage 32 using X-gal staining. Embryos from experiments performed in triplicate were analyzed at stage 32 for the location of $n$ - $\beta$-galactosidase positive cells. We counted each region where $n$ - $\beta$-galactosidase positive cells were observed densely as 1 but each region where none or several were found as 0 on the sectioned surface of the bisected embryos and the whole embryos. The sum of values of each region in the experimental tadpoles is shown in Table 2. Our analytical method was somewhat subjective, but the results from control embryos agreed well with previously published fate maps (Table 3). (D) The distribution data from Figure 1C were converted into a histogram. The histogram clearly shows that FGF signal deprivation from a single blastomere in the A-tier made most of its descendants move into the endodermal territory instead of their proper location. 
with Zeiss axoplot 2.

\section{Bead implantation assay}

Heparin coated acrylic beads (Sigma) were soaked in $50 \mu \mathrm{M}$ SU5402 or bFGF protein in $1 \times$ PBS overnight at $4^{\circ} \mathrm{C}$. BSA-soaked beads were used as a control. A bead was picked and inserted into the furrow between blastomeres using fine forceps. After healing of the opening site, the embryos were transferred to fresh $30 \%$ MMR and cultured until the appropriate stages.

\section{Results}

\section{Blocking FGF signaling caused embryonic cells to move into endodermal territory}

It has been shown that alteration of essential signaling molecules in embryonic cells can change their fate, as well as their location and the molecular nature of the cells (Fujii et al., 2002). In our study, we have investigated whether descendants of blastomeres from the 32-cell stage embryo adopted an endodermal fate following FGF signal deprivation.

In order to clarify whether the alteration of FGF signaling in embryonic cells could translocate the cells, we first traced morphological changes and locations of descendants of labeled cells in later stages following injection of a dominant negative FGF receptor (DN-FR) with $n$ - $\beta$-gal into a single dorsal blastomere of each tier of 32-cell stage embryos (Figure 1A). We found that on the whole, embryos injected with DN-FR into one cell in the A, $C$, and $D$ tiers kept their normal shape well, but injection of DNFR into a B tier blastomere gave rise to severe malformations in the embryos (Figure 1A), such as curved back and shortened tail. This result was consistent with previous observations (Amaya et al., 1993; Cha et al., 2004; Delaune et al., 2005).

The $A$ tier normally gives rise to the epidermis and brain (Dale and Slack, 1987). As expected, descendants from the A-tier, marked with $\mathrm{n}-\beta$-gal, were found in the head and anterior epidermis of the control embryos. However, $\mathrm{n}$ - $\beta$-gal co-injected with DN-FR into the same blastomeres of sibling embryos were identified in the ventral region (Figure 1A). On the contrary, when wild type FGF receptor (WT-FR) mRNA was injected into the A1 cell, its descendants were not seen in the endodermal location, but in the anterior or lateral epidermis and muscle (Figure 1C). The translocation effect following DNFR injection into presumptive epidermal cells was rescued by co-injection with wild-type FGF receptor (Figure 1C). This suggests that blocking FGF signal in blastomeres of the $A$ tier caused their descendants to move into inappropriate locations such as the endodermal region. We therefore traced whether embryonic cells could move and finally arrive in the endodermal area when their FGF signaling pathway was blocked. To test this, we co-injected GFP instead of $n$ - $\beta$-gal with DNFR into a blastomere of the A tier to follow the migration trajectory of its descendants; clones of the cell have been traced up to stage 41 (Figure 1B, Table S1). The descendants stayed in the proper place until the end of the gastrulation, as in controls, but showed active migratory movement to the ventral region during neurulation. Finally, they were found in the endodermal area at stage 26 . This implies that the fate-changed embryonic cells could migrate to the place where their new fate dictated through active cellular movement during neurulation.

For the technical control, dominant negative BMP receptor (DN-BR) alone or with DNFR was injected into the same blastomere as sibling embryos, since the role of BMP-4 signal blocking has been well documented in neuralization during early Xenopus development. In agreement with a previous report (Sasai et al., 1996), descendants of the DNBR injected blastomeres were observed in the neuroectoderm, dorsal mesoderm, and endoderm (Figure 1C, Table 2) but addition of FGF signal blocking moved most of their descendants into the posterior ventral region. Taken together, we can postulate that the translocation of the presumptive ectodermal cells into the endodermal territory following DN-FR injection is caused not by the physical stress following injection, but by fate alteration.

Descendants of D1 blastomeres injected with DN-FR were found in the proper locations (Figure 1A). However, when FGF was expressed in the D1 blastomere, its descendants were found in the lateral mesoectodermal region instead of the endoderm derivatives. Considering the aforementioned results, the inhibition of FGF signaling in the embryonic cells made them move into the endodermal area (Figure 1D, Table 3), whereas activation of the FGF signaling pathway in embryonic cells caused them to localize to the mesoectodermal area. This implies that the abdominal expansion or shrinkage of tissue in the presence or absence of FGF signaling is the morphological evidence of quantitative alteration of the endodermal mass.

\section{Blocking FGF signaling induced the presumptive mesoectodermal cells to express endodermal genes}

In order to know whether there is an alteration of 
Table 2. Effects of DNFR on the fate of blastomeres of 32-cell stage embryos.

\begin{tabular}{lccccccc}
\hline & \multicolumn{3}{c}{ Distribution of lacZ staining } & \multirow{2}{*}{$\begin{array}{c}\text { Number of } \\
\text { embryos }^{* *}\end{array}$} \\
\cline { 2 - 6 } & Epidermis & Head & Notochord & Somite & Gut & Others & \\
\hline A-tier (control) & $49(20 / 15 / 14)$ & $28(15 / 7 / 5)$ & & $20(8 / 5 / 7)$ & & $3(0 / 1 / 2)$ & $52(21 / 17 / 14)$ \\
A-tier DNFR & $13(2 / 4 / 7)$ & $8(0 / 4 / 4)$ & $3(0 / 1 / 2)$ & $9(0 / 3 / 6)$ & $63(25 / 17 / 21)$ & $4(1 / 2 / 1)$ & $71(29 / 19 / 23)$ \\
B-tier (control) & $5(1 / 2 / 2)$ & $18(6 / 5 / 7)$ & $47(18 / 14 / 15)$ & $25(9 / 11 / 5)$ & $10(0 / 8 / 2)$ & $5(1 / 0 / 4)$ & $57(21 / 16 / 20)$ \\
B-tier DNFR & & $2(0 / 1 / 1)$ & & $9(4 / 2 / 3)$ & $72(28 / 20 / 24)$ & $17(4 / 3 / 10)$ & $82(30 / 25 / 27)$ \\
C-tier (control) & $12(5 / 6 / 1)$ & $20(8 / 9 / 3)$ & $14(6 / 5 / 3)$ & $50(14 / 10 / 26)$ & $3(0 / 1 / 2)$ & $73(17 / 21 / 35)$ \\
C-tier DNFR & & $3(0 / 3 / 0)$ & $8(0 / 6 / 2)$ & $79(31 / 22 / 26)$ & $10(4 / 3 / 3)$ & $89(35 / 25 / 29)$ \\
D-tier control & & & & $92(30 / 29 / 33)$ & $8(3 / 2 / 3)$ & $94(31 / 30 / 33)$ \\
D-tier DNFR & & & & $98(31 / 36 / 31)$ & $2(0 / 0 / 2)$ & $98(31 / 36 / 31)$ \\
\hline
\end{tabular}

*Number of occurances (occurances within independent experiment divided by slash[/]); ${ }^{* *}$ Number of embryos (embryos within independent experiment divided by slash[/]).

Table 3. Effects of DNFR and/or DNBR on the fate of blastomere in A-tier.

\begin{tabular}{lccccccc}
\hline & \multicolumn{3}{c}{ Distribution of lacZ staining* } & \multirow{2}{*}{$\begin{array}{c}\text { Number of } \\
\text { embryos }\end{array}$} \\
\cline { 2 - 6 } & Epidermis & Head & Notochord & Somite & Gut & Others & \\
\hline A-tier (control) & $49(20 / 15 / 14)$ & $28(15 / 7 / 5)$ & & $20(8 / 5 / 7)$ & & $3(0 / 1 / 2)$ & $52(21 / 17 / 14)$ \\
A-tier DNFR & $13(2 / 4 / 7)$ & $8(0 / 4 / 4)$ & $3(0 / 1 / 2)$ & $9(0 / 3 / 6)$ & $63(25 / 17 / 21)$ & $4(1 / 2 / 1)$ & $71(29 / 19 / 23)$ \\
A-tier DNBR & $5(1 / 3 / 1)$ & $20(6 / 4 / 10)$ & $48(12 / 20 / 16)$ & $27(8 / 12 / 7)$ & $8(0 / 2 / 6)$ & $2(2 / 0 / 0)$ & $63(20 / 24 / 19)$ \\
A-tier DNFR+DNBR & & $2(1 / 0 / 1)$ & & $9(5 / 1 / 3)$ & $82(23 / 31 / 28)$ & $7(2 / 4 / 1)$ & $95(26 / 36 / 33)$ \\
\hline
\end{tabular}

*Number of occurances (occurances within independent experiment divided by slash[/]); **Number of embryos (embryos within independent experiment divided by slash[/]).

gene expression in early embryonic cells following FGF signal modification, we introduced beads into the presumptive meso-ectodermal area. Generally, beads have been used to introduce certain molecules into restricted areas in vivo. Heparin-coated acrylic beads were used in this study. Before implantation, we soaked the beads in SU5402 or bFGF proteincontaining solutions overnight and inserted a bead into the marginal zone through the slit using glass needles. In the case of BSA-soaked (control) bead implantation, there was no detectable abnormality in the embryos. In order to examine how the gene expression pattern is altered by FGF signaling modulation, whole mount in situ hybridization with darmin (a mid-gut marker) probe was performed. Darmin was strongly expressed in cells neighboring the SU5402 soaked bead while embryos implanted with the FGF soaked bead showed a repression of darmin expression surrounding the bead (Figure $2 \mathrm{~B})$. This is consistent with the previous finding that FGF signal blocking by injecting DNFR into the Xenopus embryos caused the expansion of the endodermin expression area (Cha et al., 2004). The introduction of a single gene determining anterior-posterior axis could change both the location and fate of the cell (Fujii et al., 2002) also supports that FGF signal blocking induced an endodermal gene where it was not supposed to be expressed. Taken together, it clearly indicates that the blockade of FGF signaling in early embryonic cells can induce endodermal gene expression as well as alter the cellular location.

\section{Inhibition of FGF signaling until stage 10.5 was required for initial endoderm formation}

The results above showed that the elimination of FGF signaling in the blastomeres made them adopt an endodermal fate. We then tried to find out when the FGF signal blocking was required for normal endodermal specification of the vegetal cells. Developmental stages were classified according to standards set by Nieuwkoop and Faber (1994). The experimental time interval (from fertilization to stage 26) was divided by two time points. The first was the midblastula transition (MBT), in which zygotic gene expression began and the other was stage 10.5 when gastrulation started. This made three time frames for our experiments. FGF signaling was blocked by soaking embryos in MMR solution containing SU5402 (Figure 3A). Based on our previous work (Cha et al., 2004), morphological alteration of the embryo was used as the criteria for the proper 
A

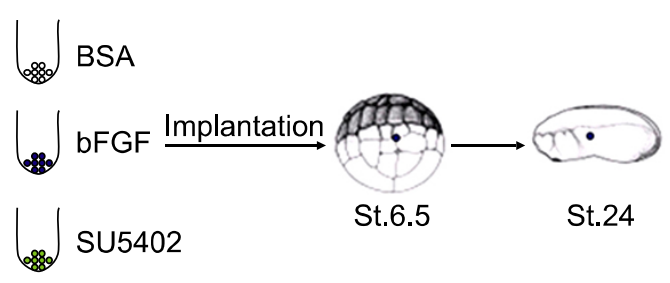

Soaked overnight at $4^{\circ} \mathrm{C}$

B

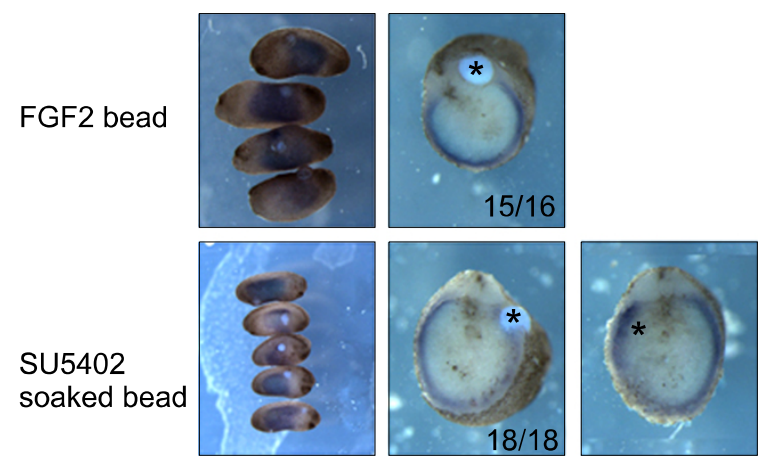

Figure 2. (A) Procedure of the bead implantation assay. (B) Bead implanted embryos were analyzed using whole mount in situ hybridization with Darmin probe, in order to label the mid-gut. An FGF-soaked bead implanted embryo showed the repression of Darmin expression around the bead (15/16 affected embryos/total embryos). However, cells neighboring the SU5402 soaked bead showed strong expression of darmin (18/18 affected embryos/total embryos). This shows that the presence or absence of FGF signaling is strongly related to the expression of endodermal genes in embryonic cells during Xenopus embryogenesis (Table S2).

blockade of FGF signaling. That is, the abdominal expansion of the embryo was counted as an increase in endoderm. A curved trunk or shortened tail was taken as a mesodermal defect. Embryos showed a prominent expansion of the abdomen accompanied by a curved trunk and shortened tail when FGF signaling was inhibited from fertilization up to stage 10.5 (Figure 3B. Group A, B and C). When FGF signaling was blocked from stages 10.5 to 26, embryos showed a contracted abdomen accompanied by a malformed back and truncated tail (Figure 3B. Group D, E and F). The malformed mesoderm and neural ectoderm sometimes caused shamed features of the abdominal expansion in the prolonged FGF signal blocking groups (Figure 3B. Group D, E and F). Taken together, the blockade of the FGF signaling pathway before stage 10.5 promoted endoderm formation but persistent FGF signaling blockade after stage 10.5 strongly repressed endodermal development.

In order to understand whether morphological changes following the FGF signal blockade were related to changes in mRNA expression level, RT-PCR analysis was performed using molecular markers for each of the three germ layers (Figure $3 \mathrm{C})$. Consistent with the morphological observation, embryos treated with SU5402 up to stage 10.5 showed increased expression of endodermin, whereas embryos treated with SU5402 after stage 10.5 showed reduced expression of endodermin accompanied with the altered expression of actin and $\mathrm{N}$-cam. Endodermin was not detected in embryos treated with SU5402 for the entire experimental period, i.e., from fertilization to stage 26. Accordingly, it is suggested that the blockade of FGF signaling before stage 10.5 is necessary for the early endoderm formation but the continuous FGF signal blocking after stage 10.5 seemed to inhibit its maintenance in the Xenopus embryos. More complicated signaling pathways suggested to be necessary for the endodermal maintenance after stage 10.5 and be identified through further studies.

We performed tissue recombination experiments to understand the effects of FGF signal blockade on endodermal differentiation. Our results showed that continued FGF signal blocking after MBT did not induce more differentiated endodermal markers such as XIhbox8 and IFABP in the conjugates of the vegetal and animal caps while the conjugates cultured in NAM solution expressed them (Figure 3D). This was consistent with a recent report showing that blockage of FGF signaling significantly reduced maturation of visceral endoderm in mouse embryonic stem cells (Esner et al., 2002).

\section{Discussion}

We showed that the blocking of FGF signaling caused descendants of the presumptive ectodermal cells, whose fate would not normally be endoderm, to distribute into the gut and the abdominal region of tailbud stage embryos. Cellular trans-location to the abdominal region caused by the FGF signaling blocking, which is rescued simply by coinjection of wild type FGF receptor. Presumptive ectodermal cells expressed endodermal genes in situ when FGF signaling was blocked. It suggests that FGF signaling blocking in the early embryonic cells can alter the fate of their descendants. FGF signaling blocking before stage 10.5 caused abdominal expansion and the increased endodermal gene expression suggesting that FGF signal blocking may play critical roles at the very beginning step of the endoderm formation. In vertebrate embryos, the spatially restricted expression of specific maternal 
A

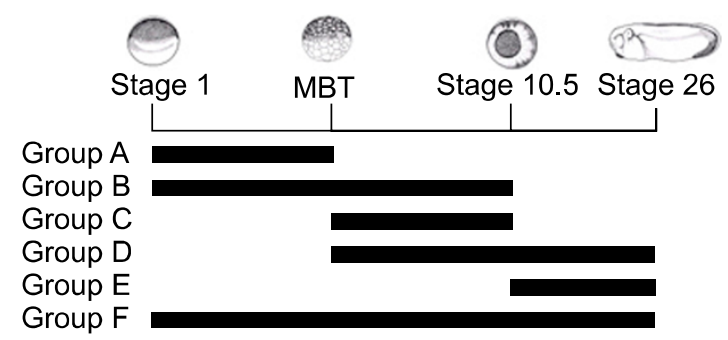

C

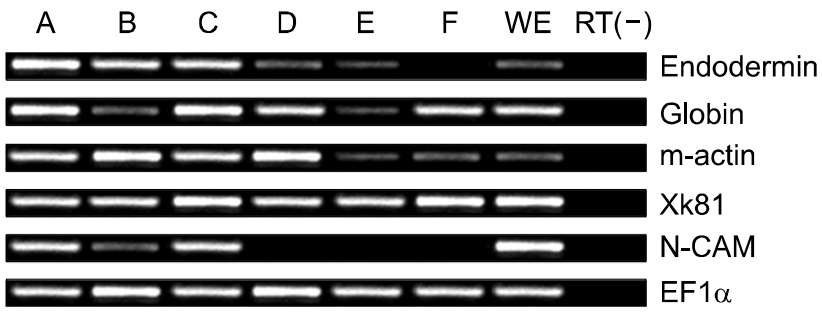

B

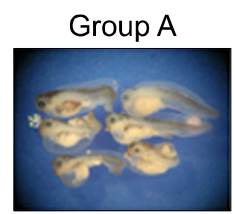

Group D

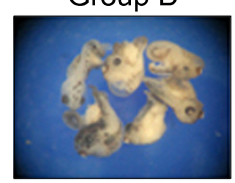

Group F

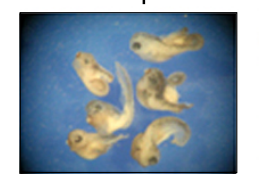

Group B

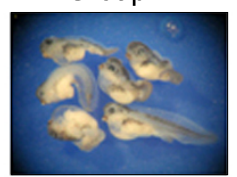

Group E

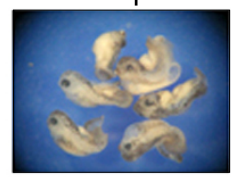

Control

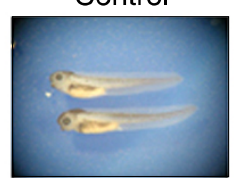

Group C

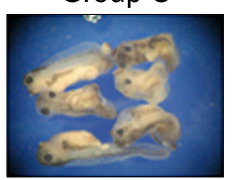

D

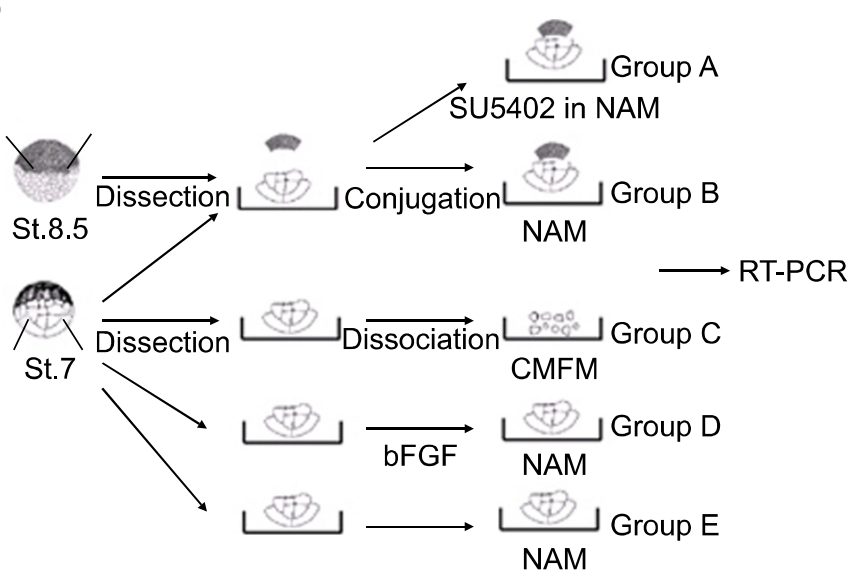

Endodermin

Xlhbox8

IFABP

Figure 3. (A) Schematic diagram of the time frame of SU5402 treatment of Xenopus embryos. (B) Morphology of embryos treated with SU5402 according to the designed time frame. Most of the embryos that were treated with SU5402 before stage 10.5 (groups A, B, and C) showed an expanded abdominal region. However, a curved back and shortened tail were seen in the group treated with SU5402 after stage 10.5 (group E). (C) Gene expression pattern of embryos treated with SU5402 according to the time frame. Expression of endodermin appeared to be normal in the case of FGF signal deprivation in embryos from fertilization to stage 10.5. However, its expression was abruptly decreased when the FGF signal was blocked after stage 10.5. This shows that FGF signal blockade before stage 10.5 is critically important for proper endoderm formation but persistent blocking of FGF signal after stage 10.5 inhibited proper endoderm formation. When the FGF signaling was blocked after stage 10.5, neural induction was inhibited as shown (Group D, E, F), and the expression of actin, a general mesodermal marker, was also reduced (group E, F). (D) The future endodermal region of the vegetal hemispheres was dissected from embryos at MBT and conjugated with animal cap explants. The conjugated explants were cultured with FGF signal blocking until stage 30. As shown in Group A, the expression of the general endodermal marker endodermin was increased by RT-PCR analysis but the expression of endodermal organ specific markers such as XIhbox8 and IFABP were not observed when compared with conjugated explants cultured without SU5402. The RT-PCR results showed that the explants remained in the initial stage of the endodermalization instead of differentiating into the specific organ cells as shown at Group B.

genes predicts the establishment of the three germ layers and the axis formation. Significance of the spatially restricted expression of a certain signaling is already acknowledged in cases of BMP-4 signaling in differential specification of neuroectoderm and ectoderm and Wnt signaling in dorsoventral pattern formation (Schohl and Fagotto, 2003; Delaune et al., 2005). Similarly, our results strongly support that the vegetally restricted blocking of FGF signaling may also play critical roles for differential development of mesoderm and endoderm from early embryonic cells. In this context, deprivation of FGF signaling must be considered as much important as presence of FGF signaling for proper germ layer specification particularly, endodermmesoderm formation.

FGF signal blockage has been reported to occur in the vegetal region of the Xenopus embryo. For instance, the expression level of the FGF receptor in the vegetal hemisphere is extremely low as 
compared with the animal hemisphere. Furthermore, Xtsulf1, a maternal transcript coding for $\mathrm{N}$-acetylglucosamine-6-sulfatase that modifies HSPG sulfation and negatively regulated FGF signaling, is present in the vegetal hemisphere (Freeman and Pownall, 2004 10th international Xenopus meeting). This suggests that the uneven distribution of active FGF signaling is already established in fertilized eggs.

In conclusion, blocking FGF signaling in vegetal cells between fertilization and stage 10.5 makes them adopt an endodermal fate and localize to vegetal region. It indicates that the restricted FGF signal elimination in the vegetal hemisphere is one of the absolute requirements for initial endoderm formation in Xenopus embryos. However, the persistent inhibition of FGF signaling after stage 10.5 disturbed endodermal differentiation.

\section{Acknowledgements}

This work was supported by the Korea Research Foundation Grant funded by the Korean Government (MOEHRD, Basic Research Promotion Fund) (R04-2004-000-10120-0, KRF: E00032) and by the Brain Korea 21 Project in 2006 (Sang-wook Cha).

\section{References}

Agius E, Oelgeschlager M, Wessely O, Kemp C, De Robertis EM. Endodermal Nodal-related signals and mesoderm induction in Xenopus. Development 2000;127:1173-83

Amaya E, Stein PA, Musci TJ, Kirschner MW. FGF signalling in the early specification of mesoderm in Xenopus. Development 1993;118:477-87

Cha SW, Hwang YS, Chae JP, Lee SY, Lee HS, Daar I, Park MJ, Kim J. Inhibition of FGF signaling causes expansion of the endoderm in Xenopus. Biochem Biophys Res Commun 2004;315:100-6

Ciruna B, Rossant J. FGF signaling regulates mesoderm cell fate specification and morphogenetic movement at the primitive streak. Dev Cell 2001;1:37-49

Clements D, Friday RV, Woodland HR. Mode of action of VegT in mesoderm and endoderm formation. Development 1999;126:4903-11

Clements D, Woodland HR. VegT induces endoderm by a self-limiting mechanism and by changing the competence of cells to respond to TGF-beta signals. Dev Biol 2003;258: 454-63

Cornell RA, Musci TJ, Kimelman D. FGF is a prospective competence factor for early activin-type signals in Xenopus mesoderm induction. Development 1995;121:2429-37

Delaune E, Lemaire P, Kodjabachian L. Neural induction in Xenopus requires early FGF signalling in addition to BMP inhibition. Development 2005;132:299-310
Dale L, Slack JM. Fate map for the 32-cell stage of Xenopus laevis. Development 1987;99:527-51

Dale L. Vertebrate development: Multiple phases to endoderm formation. Curr Biol 1999;9:R812-5

Esner M, Pachernik J, Hampl A, Dvorak P. Targeted disruption of fibroblast growth factor receptor-1 blocks maturation of visceral endoderm and cavitation in mouse embryoid bodies. Int J Dev Biol 2002;46:817-25

Fujii H, Nagai T, Shirasawa H, Doi JY, Yasui K, Nishimatsu $\mathrm{S}$, Takeda H, Sakai M. Anteroposterior patterning in Xenopus embryos: egg fragment assay system reveals a synergy of dorsalizing and posteriorizing embryonic domains. Dev Biol 2002;252:15-30

Heasman J. Patterning the Xenopus blastula, Development 1997;124:4179-91

Heasman J. Maternal determinants of embryonic cell fate. Semin Cell Dev Biol 2006;17:93-8

Hilton E, Rex M, Old R. VegT activation of the early zygotic gene Xnr5 requires lifting of Tcf-mediated repression in the Xenopus blastula. Mech Dev 2003;120:1127-38

Kofron M, Wylie C, Heasman J. The role of Mixer in patterning the early Xenopus embryo. Development 2004;131:2431-41

Mohammadi M, McMahon G, Sun L, Tang C, Hirth P, Yeh BK, Hubbard SR, Schlessinger J. Structures of the tyrosine kinase domain of fibroblast growth factor receptor in complex with inhibitors. Science 1997;276:955-60

Munoz-Sanjuan I, H-Brivanlou A. Early posterior/ventral fate specification in the vertebrate embryo. Dev Biol 2001; 237:1-17

Nieuwkoop PD, Faber J. Normal Table of Xenopus laevis (Daudin), 1994, GARLAND PUBLISHING, New York\&London, USA

Patient RK, McGhee JD. The GATA family (vertebrates and invertebrates). Curr Opin Genet Dev 2002;12:416-22

Sasai Y, Lu B, Piccolo S, De Robertis EM. Endoderm induction by the organizer-secreted factors chordin and noggin in Xenopus animal caps. Embo J 1996;15:4547-55

Schohl A, Faggotto F. A role for maternal beta-catenin in early mesoderm induction in Xenopus. EMBO J 2003;22:3303-13

Sive HL, Grainger RM, Harland RM. Early Development of Xenopus laevis: A Laboratory Manual, 2000, Cold Spring Harbor Laboratory Press, Cold Spring Harbor, New York, USA

Stemple DL. Vertebrate development: the subtle art of germ-layer specification. Curr Biol 2001;11:R878-81 Vincent SD, Dunn NR, Hayashi S, Norris DP, Robertson EJ. Cell fate decisions within the mouse organizer are governed by graded Nodal signals. Genes Dev 2003;17:1646-62

Wardle FC, Smith JC. Transcriptional regulation of mesendoderm formation in Xenopus. Semin Cell Dev Biol 2006; 17:99-109

Xanthos JB, Kofron M, Wylie C, Heasman J. Maternal VegT is the initiator of a molecular network specifying endoderm in Xenopus laevis. Development 2001;128:167-80 